\title{
COVERT HOMICIDE: WHEN SIDS IS NOT SIDS, REASONS FOR THE MISSED IDENTIFICATION
}

\author{
Steven A. KOEHLER ${ }^{1}$, Karen M. APPLEGATE ${ }^{2}$
}

${ }^{1}$ Department of Epidemiology University of Pittsburgh PA USA

${ }^{2}$ University of Pittsburgh Medical Center Pittsburgh, PA USA

Steven A. Koehler

University of Pittsburgh Graduate School of Public Health Department of Epidemiology 130 De Soto St.

Pittsburgh PA, 15261 USA

sakoehler100@hotmail.com

Tel.: + 14124872531

Fax.: + 14126478445

Received: November 11, 2012

Accepted: February 13, 2013

Copyright $(\subset) 2013$ by

University Clinical Center Tuzla. E-mail for permission to publish: paediatricstoday@ukctuzla.ba
The sudden and unexpected death of a healthy infant requires a forensic and legal investigation. If the forensic investigation fails to identify a significant cause for the death, the death is labeled Sudden Infant Death Syndrome (SIDS). Among all the possible causes for the death murder is rarely considered in the differential diagnosis. Studies have reported that as many as $20 \%$ of SIDS deaths are in fact homicide. This paper will examine factors within the medicolegal and the law enforcement communities that seriously affect the detection of infant deaths that masquerade as SIDS. In addition, it examines how the preconceived portrayal of motherhood by society and societal pressures impede the detection of mothers that murder their children. Among the 2,500 SIDS deaths annually in the U.S., it is estimated that between 25 to 250 of these infants are killed by their mothers. In order to accurately ascertain the number of infants that are murdered requires a significant shift in the mindset of all those involved in the investigation. This will result in justice for the infant, the future protection of other infants, and provide a more accurate representation of infants that truly die from SIDS. Conclusion - Therefore, to ensure that the true cause of the sudden and unexpected death of an infant is ascertained, all infant deaths must undergo a complete forensic and police investigation. In addition, this practice will ensure the integrity and accuracy of cases designated as SIDS.

Key words: Homicide - SIDS - Death investigation - Forensics

\section{Introduction}

The sudden and unexpected death of a health infant is a shocking event. The scene is emotionally charged, filled 
with panicstricken parent(s), Emergency Medical Service (EMS) personnel conducting a rapid assessment of the infant, and the police trying to maintain some sense of calm at the residence. If the infant is pronounced dead, the atmosphere becomes even more stressful and at times even hostile, especially when the parent(s) are informed that the law requires a forensic medical investigation to be conducted by the Medical Examiner's or Coroner's office $(\mathrm{ME} / \mathrm{C})$. The residence has now turned into a death scene where EMS personnel must protect the infant and the integrity of the death scene until the arrival of the death investigators from the $\mathrm{ME} / \mathrm{C}$ office. The role, training, and management of the scene by EMS and/or first-response teams, involving a sudden and unexpected infant death at a residence are outlined in detail by Hymel (1). The police at the scene must begin their independent investigation. They must first remove the parent(s) and other family members from the infant and the death scene. Second, they must separate the parents to conduct separate interrogations. They begin by informing the parent(s) that the law mandates that law enforcement and the $\mathrm{ME} / \mathrm{C}$ office must investigate all sudden and unexpected deaths, especially those involving infants. However, the presence of police cars and the arrival of a van with the letters MEDICAL EXAMINER gives the appearance that something suspicious has occurred.

There is a tendency for those involved in the investigation, such as law enforcement, the courts, the forensic community, and the public, to treat this type of death differently from other sudden unexpected deaths. Compare this to the discovery of a young male discovered dead in an alleyway late at night. This death would be investigated as a possible homicide and all efforts would be employed to support that opini- on. In contrast, all those involved with the investigation of an infant's death are of the mindset that the death was natural in nature. Those involved in the investigation of an infant death seem to take the view that these deaths should be classified as natural unless there is compelling evidence to the contrary (2). This mirrors the legal concept that a person is presumed innocent until proven guilty beyond a reasonable doubt. However, this form of thinking has no place in science or in the investigation of a death. The scientific approach requires that all possible causes of death must be considered and based on a review of all the available evidence and arrive at the most probable cause of death (2). Conducting any investigation with the pre-determined mindset that the most likely cause of death is natural will result in the following: missing or overlooking evidence indicating that the death was really a homicide, the conduct of weak or ineffective questioning of the most likely suspects, the parent(s), and an unwillingness to bring formal charges against the mother out of fear of public backlash. These factors can result in missing covert homicides that present as SIDS deaths. The medical literature contains a number of well-validated reports of child abuse and infanticide, intentional suffocation presenting as apparent life threatening events (ALTES) and/or apparent SIDS (1). In addition, the lay press is filled with cases of murder initially diagnosed as SIDS $(1,3)$. The exact number of homicides that go unrecognized, where the deaths are mistakenly attributed to other causes, such as SIDS, is difficult to ascertain (4).

This paper will examine three factors that limit or hamper the detection of murder of infants masquerading as SIDS. First, we will define SIDS and the specific protocol required to classify an infant's death as SIDS. Second, we will examine the limitations of the forensic examination to diffe- 
rentiate SIDS from certain types of murder. Third, we examine the role of law enforcement and the criminal justice system in these types of investigation and fourth, the omnipresent influence that society gives to the death investigation. Finally, we present an estimate of the number of covert murders masquerading as SIDS.

In this paper, a Pubmed search was conducted using the keywords: Murder, SIDS, and Masquerading, to identify related articles. In addition, a web search was conducted using similar keywords.

\section{Classification of a SIDS death}

It is important to understand the current definition of SIDS. The classic SIDS death involves a relativity healthy infant that is fed normally before being placed in bed to sleep; no crying is heard during the night, the next morning the infant is found unresponsive in the same position in which it was placed to sleep (1). The main characteristics of these infants include: age between 1-12 months old with the majority (90\%) occurring between 2-6 months of age, sex - predominantly male, race - black with a 2-3 times higher rate compared to whites, sleeping position -prone sleeping position, seasonality - more deaths during the winter months, infant health - no major illness, death - unwitnessed, sudden and unexpected, maternal characteristics - young, single, poor, and limited or no prenatal care $(1,5,6)$. SIDS is the leading cause of death among infants between the ages of 1 month and 1 year of age in the developed counties (7). In the United States, there are 2,500 SIDS deaths annually, accounting for $22 \%$ of all postnatal deaths (7). The majority of SIDS deaths are "true" SIDS, however, some are murder. A number of scientific studies have described cases of infant deaths that were attributed to SIDS but subsequent investigation showed they were in fact infanticide by smothering (8).

A specific protocol must be followed to classify an infant's death as SIDS. First, a thorough death scene investigation must be conducted. This investigation should include a detailed examination of the infant's sleeping environment. Second, a forensic autopsy is undertaken that includes an external and internal examination of the body, performed by a forensic pathologist. This examination should include full body x-rays, histological and toxicological analysis, and genetic screening. Third, a detailed review of the past medical history of the infant and mother including prenatal care, characteristics of the delivery and wellness visits must be done. If all three levels of the investigation fail to identify a significant cause for the death of the infant, then and only then can the death be signed out as SIDS, with the manner of death as natural. SIDS is a diagnosis of exclusion, therefore all possible causes of the death must be fully investigated and excluded for it to be a "true" SIDS death. This scenario is in an ideal world. In some locations even within the United States and in many other parts of the world, the investigations fall far short of these basic standards (5). Figure 1 shows the steps of the forensic investigation. If all three phases are negative, the infant's death should be labeled as SIDS. If the results from any phase of the investigation reveal questionable or inconsistence findings, the manner of the infant's death on the certificate should be designated "Undetermined". If sufficient evidence is present to support a non-natural death, it should be designated as Accident or Homicide. If the infant's death does not undergo all three phases, that infant's death cannot be classified as SIDS. 


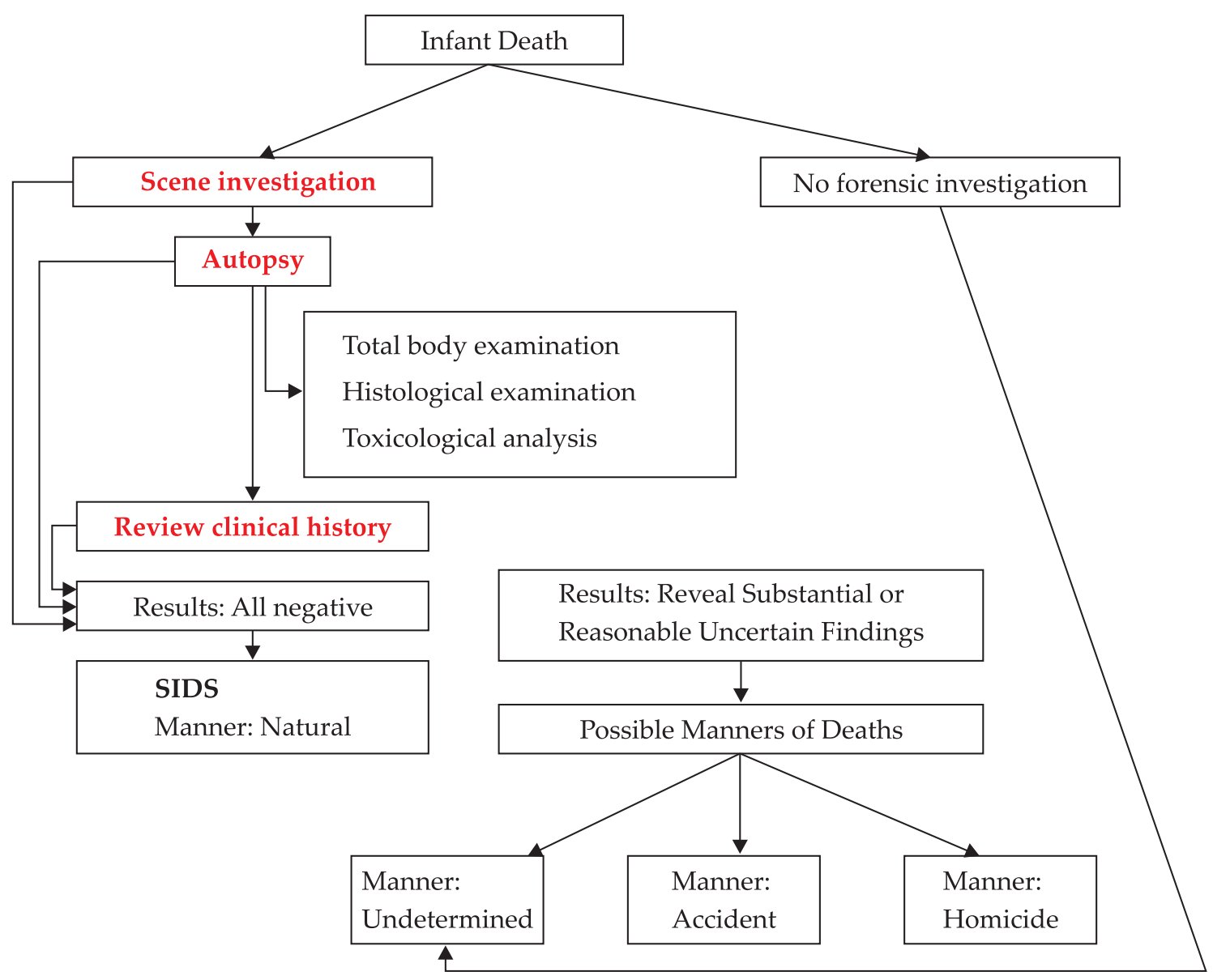

Fig. 1 Infant death investigation protocol

\section{The forensic investigation}

The bias towards a natural death appears to start early, well before any form of investigation is conducted. Almost immediately, the EMS pronouncing the infant dead designates the death as a SIDS death. This initial label impacts future investigations, because the death will be seen as a natural death. The law requires that when an infant dies suddenly and unexpectedly the death must be investigated by the ME/C office (5). The forensic investigation is composed of two phases: the death scene investigation and the post mortem examination.
The death scene investigation, even when conducted in great detail, offers little, if any evidence that would indicate any foul play occurred. The infant's room and surroundings typically present as normal. The scene investigation is critical to eliminate evidence to support accidental suffocation due to bedding, defects in the crib, or other mechanisms that could have caused accidental asphyxiation. During this phase of the investigation, the parent(s) are interviewed by the death investigators regarding the events leading up to the discovery of the infant. While the majority of infant deaths occur while 
under the care of the mother, a number of cases involved death while being watched by the mothers' boy-friend, baby-sitters ( 9 , 10), or other family members. Part of the investigation includes the completion of the CDC developed "SIDS Data Collection Form" (11). This form is designed to collect well-known risk factors and behaviors associated with SIDS. While the information collected is critical in the discovery of risk factors associated with SIDS, it also has a prejudicial effect. It reinforces the template that the death was due to SIDS. The SIDS Data Collection Form is not a tool designed to investigate homicides.

The second part of the forensic investigation is the complete postmortem examination. The examination of the infant, even under the best circumstances, will typically fail to reveal any clear and definitive evidence indicating that a murder has occurred. The reason for this is that the most common method used to murder an infant masquerading as SIDS is suffocation (8). This mechanism of death offers a great challenge to forensic investigators because of the difficulty in differentiating suffocation from SIDS. There are very few external signs to make this differentiation (12-22). The two frequent methods used to suffocate an infant involve using the hand or by the use of a pillow. Suffocation by hand requires applying a minimal amount of force to occlude the mouth and nose and results in no specific forensic evidence of the mechanism (23). It is also well understood that smothering an infant with a pillow also leaves no clear forensic evidence of the act (23).

The autopsy also includes histological examination of the internal organs and toxicological analysis of body fluids. The required histological examination is not without utility. Some studies have shown that histological examinations of pulmonary tissue have differentiated SIDS from smothering.
Important histological markers have been reported for the diagnosis of death due to asphyxia caused by obstruction of the respiratory passage. The detection of these histological markers would strengthen the diagnosis that a death was caused by suffocation (23). The toxicological analysis is also critical in identifying infants that died from an accidental overdose of prescription medication or from deliberate poisoning (1).

Some have suggested that because the forensic investigation cannot definitely prove SIDS, or murder masquerading as SIDS, they are not necessary, therefore the family should be spared the trauma of an autopsy. However, this rationale should not be used to justify omitting a complete forensic autopsy, for several reasons. There are numerous ramifications associated with not conducting a forensic investigation and autopsy. First, based on the standard definition of SIDS, an investigation and forensic autopsy are required to certify an infant's death as SIDS. Without an autopsy and scene investigation, the diagnosis of SIDS cannot be assumed with sufficient certainty (23) and cases of child maltreatment can go undetected (1). Second, the autopsy is critical in discovering congenital conditions, metabolic disorders, such as inborn errors of fatty acid oxidation, and other rare conditions that were missed during life. These disorders have minimal symptoms and can mimic suffocation or SIDS (24). In addition, the identification of medical or genetic conditions can save the lives of future offspring at risk of death. Third, public health threats can go undetected, product-safety issues remain unidentified and inadequate medical care can go unreported (1). Finally, the progress in the understanding of the etiology of SIDS and other causes of unexpected infant deaths are delayed (1).

Studies have shown that the death scene investigation and the adherence of the 
standard autopsy protocol in cases of SIDS vary greatly across the county (7). The pathologists conducting the autopsy have great discretion when designating the cause and manner of death. It has been reported that some pathologists under diagnosis SIDS, while others apply the diagnosis too liberally (25). Reasons for this include a misguided $\mathrm{ME} / \mathrm{C}$ officer, feeling that the parent(s) have suffered enough and there is no need to dissect the infant. In many parts of the world, there is a total lack of any form of death investigation. This is primarily due to a lack of forensic pathologists to conduct proper forensic investigations, therefore infant deaths are simply designated as SIDS.

One of the clearest markers to raise a high level of suspicion of a covert homicide is recurrent SIDS involving the same mother. Many studies have stated that recurrent infant deaths in one family are homicides (16, 18, 21, 26-31). In 1989, two well-known U.S. forensic pathologists, DiMaio and DiMaio, developed a maxim relating to multiple SIDS deaths within the same family. The first infant that died can be classified as SIDS, a second death to the same mother should be classified as an "Undetermined," however if a third infant dies suddenly and unexpectedly, that death is a Homicide and the first two deaths should undergo a through second examination and review (32). In Europe, this policy is known as the Meadow's Law, which states that one SIDS death is tragic, two are suspicious, and until the contrary is proven, the third SIDS like death is murder (33). As outlined above, it is extremely difficult, if not impossible, to distinguish between SIDS and suffocation at autopsy, however, there are certain circumstances that could indicate the possibility of intentional suffocation. These included the following: (1) Recurrent cyanosis, apnea, or ALTE's, occurring only while in the care of the same caregiver, (2) Previous unexpected or unexplained death of one or more siblings, (3) Simultaneous or nearly simultaneous death of twins, (4) Previous deaths of infants unrelated to the caregiver, and (5) Evidence of previous pulmonary hemorrhages $(1,34)$.

In many states, Child Death Review Teams have been established to review all deaths under the age of 18 within a set region. These committees are composed of representatives from the $\mathrm{ME} / \mathrm{C}$ office, law enforcement, Emergency Medical Services, Prosecutors, Emergency Room Physicians, Pediatricians, and the Child Welfare/Child Protective Service. The role of these committees is to review all deaths and ensure that deaths caused by abuse are not missed, and that surviving and subsequent siblings are protected (1). Having served on a Child Death Review Team, I have seen how important data from one agency were not always shared or were inaccessible to other agencies during the investigation. I propose nationalization of the current Sudden Unexpected Infant Death Case Registry (SUID-CR) pilot program, developed by the CDC (35). The current program includes death certificates, $\mathrm{ME} / \mathrm{C}$ data, law enforcement records, medical records (birth certificates, obstetric records), and social services records, but it currently only covers less than a dozen states and is state-based. One of the weaknesses of the current system, from an investigative perspective, is linkage of related infant deaths. A national surveillance system must be created that assigns each mother, that has had a sudden and unexpected infant death, a unique identification number that is unaffected by name change or relocation, and linking all 50 states. Therefore, if a mother has a second SIDS like death in a different state from the first one, those investigating the death will be able to search the registry and locate that first death and the circumstances surrounding that death. 


\section{Law enforcement and the criminal justice system}

Law enforcement conducts its own independent investigation into the infant's death. The attitude among law enforcement investigators and the prosecutors that parents are not capable of killing their offspring is the second factor that may influence the ability to detect if the infant was the victim of homicide. This attitude first manifests itself by the lack of investigators conducting an aggressive investigation and the almost gentle treatment of the parent(s). Some may feel uncomfortable aggressively investigating a mother that has just lost her infant, as a true suspect. However, detectives do not appear to have any difficultly when they question an $\mathrm{X}$-husband when his young, attractive $\mathrm{X}$-wife has been discovered brutally killed in the couple's former residence.

The criminal standards required to obtain a criminal conviction include three basic elements: Means, Motive, and Opportunity. Applying these three standards, it is clear that the mother must be treated as the prime suspect. Typically, the mother spends the greatest amount of time with the infant, often alone. The means of killing, typically by suffocation, requires minimal planning and requires either a pillow or hands to facilitate. As stated earlier, this mechanism leaves little or no forensic markers. Motive includes poverty, the stress of a crying infant, and immaturity, which have all been shown as triggers for infant homicide (36).

The courts are also walking a fine line when it comes to infant deaths. Prosecutors are reluctant to arrest, handcuff, and charge a mother with murder. Their motives for resistance are threefold. First, the forensic evidence is extremely weak and a far cry from "beyond a reasonable doubt." Second, persecutors are unanimously aware that the only chance of obtaining a murder conviction is if the mother confesses (37). Third, becau- se individuals such as the District Attorney are in an elected position, they may fear public backlash if they bring charges again a mother without strong evidence and hoping for court room confusion. Brian Holmgren, the assistant district attorney general in Nashville, Tennessee, summed it up this way: "If you don't have a confession or some prior evidence suggesting a pattern of behavior on the part of the caretaker, it's difficult to obtain a conviction" (38).

This resistance by prosecutors is exemplified by the 1996 death of a toddler named Dreahon Maninor in Camden County, USA. The medical examiner found the child covered with scars and evidence of strangulation, and ruled the death a homicide. The prosecutors said he found no evidence of foul play and believed the death was an accidental overdose (29).

A vulnerable, defenseless, and helpless infant has no advocate to seek justice if the individuals charged with the investigation and enforcement of laws fail to approach the case open minded, which includes allowing for the possibility of murder.

\section{Society}

The third factor affecting the overall investigation is societal pressure. Societal portrayal of mothers as protectors of their children and the public cannot accept the image of a mother as the killer of her offspring. The medical community, and especially those health care workers that interact with children, also find it difficult to consider the possibility that parents are capable of smothering their children (40-42).

This societal impression of motherhood has infiltrated the legal and forensic investigation of infant deaths prematurely labeled as SIDS. A 1978 pediatrics textbook on SIDS, listing possible etiology, did not make any mention of fatal abuse within the differential 
diagnosis (43). Law enforcement would be portrayed by the media and the community as being insensitive by arresting, detaining, jailing, and interrogating a mother that has just lost her infant. The district attorney's office would hesitate to file charges against the mother without clear tangible evidence that a crime has occurred, due to outside public pressure. The forensic community is sheltered and protected from criticism by their legal requirement to conduct a death investigation. However, due to the limits of forensic science, it can offer limited evidence to support murder. Primarily due to the difficulty in differentiation of SIDS from murder, the forensic pathologists tends to choose the less controversial diagnosis of SIDS over the one of murder.

\section{The reality of infant murder}

While most individuals want to believe that infant homicides do not exist, irrefutable evidence has forced the legal, forensic and society to face the fact that mothers are not only capable of killing their infants, but some are also serial infant killers. In these cases, all infant deaths were classified as SIDS it was not until the mother later confessed that the cause of death was changed to homicide. These types of cases serve to highlight three truths. First, a number of infants that are murdered are inaccurately diagnosed as SIDS. Second, mothers are capable of murdering their infants. Third, the failure to aggressively investigate all infant deaths that are sudden and unexpected results in a killer being free to kill future infants, and there is no justice for the helpless victim.

The exact number of infanticides labeled as SIDS is difficult to ascertain for the following reasons: (1) currently murder by suffocation is indistinguishable from SIDS at the pathological level, (2) a lack of detailed and aggressive investigation (forensic and law enforcement), (3) the perpetrator acts in secret, (4) the ability of the murders to play the victim and play on the sympathy of society, and (5) SIDS is an acceptable alternative explanation for the circumstances (4).

A number of studies have attempted to estimate the numbers of murders within cases signed out as SIDS. Most studies have estimated that between $1 \%$ to $5 \%$ of SIDS are covert murders (44), a few place the prevalence at $10 \%$ (2), or some as high as $20 \%$ (45). The AAP estimated that $<5 \%$ of SIDS are due to abuse (44). It has been estimated that between $0.6 \%$ (46) to $10 \%$ (45) of deaths that are sudden and unexpected are due to suffocation. Studies have reported that $2 \%$ of SIDS are homicides due to suffocation $(18,19,23,29)$. Studies conducted in 1986 and 1993 concluded that 2/5 of repeat SIDS probably resulted from covert homicide (2). The largest series of covert murders, originally ascribed to SIDS, were the studies conducted by Meadow in 1990. The report examined 81 natural infant deaths with 42 SIDS later determined to be homicide (4). If a homicide goes undetected, there is a tendency for it to recur within the same family (27). The most infamous case was that of Waneta Hoyt from New York. His woman lost five of her children between 1965 and 1971. All five deaths were ruled SIDS by the Medical Examiner. Eventually, she confessed that she had murdered all five babies because she was tired of their crying (47). A baby sitter can also be a serial infant murderer. In 1997 a baby sitter was convicted of murdering two infants in her care by smothering. Both deaths where listed as SIDS. The conviction was only possible on the basis of a confession obtained during interrogation of the woman (48). Based on these estimates, within the U.S. with the current level of investigation between 25-250 cases of murder are missed every year. In addition, it places subsequent children at great risk of death. 


\section{Conclusion}

The discovery of a dead infant without any visible sign of trauma or a past medical history to explain the death must undergo an investigation by law enforcement and the medicolegal establishment. As a mysterious death, all manners and causes of death must be given equal weight and likelihood. The current investigation is seriously handicapped by an atmosphere of emotions interfering with what should be a neutral investigation. These limits must be removed from the investigation in order to discover the facts surrounding the death and the "true" cause of death of the infant. The possibility of murder as a differential diagnosis as to the etiology of sudden unexpected death of infant must always be considered. Today the existence of infanticide and the fact that a number of infants that present as SIDS have in fact been murdered has been well established. For an excellent review of infanticide, see the review conducted by Pitt (36). This paper has highlighted some of the main barriers that have the ability to limit the detection of infants that are murdered. These three areas are rooted in forensic science, criminal justice, and society, affecting the detection of infants that were murdered at the hand of their parents. The first is the science of forensic pathology. It is currently limited in its ability to positively identify markers of infant suffocation that are "beyond a reasonable doubt." The science of forensic pathology must continue to make advances to identify markers of suffocation. The limitations of forensic science have shifted the burden of proof of murder to law enforcement. Law enforcement must interrogate the caregivers at the same level of intensity as any other prime suspect in a death investigation. The medical literature is filled with cases of mothers that are convicted of murder after a death that was originally diagnosed as SIDS. In 1995, a mother was convicted of murde- ring five of her children by suffocation; all five deaths were listed as SIDS (8). This case highlights the failure of law enforcement to consider or recognize fatal child abuse (8). Mothers that murder their infants will not confess willingly, but intense questioning is required. Some may feel that it might be unthinkable to impose this level of interrogation upon a mother who has just lost her infant. However, it is even more unthinkable to allow an individual to go free and to kill again. The literature has shown that women that successfully kill their first infant go on to kill future offspring and even those they adopt. A 2004 report reported a young mother that smothered two of her infant daughters and both deaths where signed out as SIDS. The forensic pathologist conducting the first autopsy recommended that law enforcement conduct further investigation, but the public prosecutor failed to investigate further. It was not until the third infant death that the police conducted an extensive investigation. The investigation resulted in the mother confessing to smoothing the infants with a pillow (23). A skilled interrogation after the first death might have discovered what really transpired surrounding the first death and would have prevented future deaths. The mother's motive for the infanticide was that caring for the infant was too strenuous (23). The role of the courts and prosecution must change. An increase in the number of formal charges of murder and successful convictions for infant murder requires the willingness of prosecutors to understand that mothers are in fact committing murder, but also the current limits of proving such acts. Finally, society needs to realize that their portrayal and negative pressure is allowing mothers' to commit murder and avoid prosecution for their actions in a very tangible way.

In summary, a neutral investigation, where all possible explanations are considered, will result in cases that are classified as SIDS 
representing "true" SIDS deaths, prevent future murders from occurring and give a better understanding of the profile of women that commit infanticide. The accurate identification of murders masquerading as SIDS also has an epidemiological impact on the study of SIDS. The removal of nonSIDS from the analysis of SIDS cases will

\section{References}

1. Hymel KP. Distinguishing Sudden Infant Death Syndrome from Child Abuse Fatalities. Pediatrics. 2006;118:421-7.

2. Bacon C. Repeat sudden unexpected infant deaths. Lancet. 2005;365(9465):1137-8.

3. Baby Docs: Possible Murder in SIDS Cases. Lindsey Tanner. ABC NEWS February 5, 2013. Available at: http://abcnews.go.com/Health/ story?id $=117648 \&$ page $=1$

4. Levene S, Bacon CJ. Sudden unexpected death and covert homicide in infancy. Arch Dis Child. 2004;89:442-7.

5. Koehler SA. Forensic Epidemiology. Boca Raton: CDC Press, 2010.

6. Kairys SW. Distinguishing Sudden Infant Death Syndrome from Child Abuse Fatalities. Am Acad Pediatrics. 2001;107:437-41.

7. Hauck FR, Tanabe KO. International trends in SIDS: Stabilization of rates requires further action. Pediatrics. 2008;122:660-6.

8. Truman TL, Ayoub CC. Considering Suffocation Abuse and Munchausen by Proxy in the Evaluation of Children Experiencing Apparent LifeThreatening events and Sudden Infant Death Syndrome. Child Maltreatment. 2002;7:138-48.

9. Montco D.A.: Baby's '92 Death Murder, Not SIDS. Stephanie Farr. Philly News. August 23, 2011. Available at http:/ / www.phillyburbs.com news crime babysitter-charged-in-infant-death article_6a63b632-d2fc-5fbb-a329-39f0147bbfae html

10. Baby Sitter Charged in " 92 Infant Death. Margaret Gibbons. Phillyburbs. August 23 2011. Available at http://www.phillyburbs.com/news/crime/babysitter-charged-in-infant-death/article_6a63b632d2fc-5fbb-a329-39f0147bbfae.html result in a more accurate representation of the syndrome and allow for more accurate identification of risk factors and behaviors associated with "true" SIDS deaths.

Conflict of interest: The authors declare that they have no conflict of interest. This article was not sponsored by any external organisation.

11. CDC's Sudden Unexpected Infant Death Initiative. Sudden Unexpected Infant Death and Sudden Infant Death Syndrome. Available at: http:// www.cdc.gov/sids/suidabout.htm

12. Berry PJ. Intra-alveolar hemorrhage in SIDS: A cause for concern? J Clin Path.1991;52:553-4.

13. Du Chesne A, Bajanabowski T, Brinkmann B. Spurearmae Totungsdelite an Kindern. Arch Krimino. 1997;21-6.

14. Emery JL. Child abuse, sudden infant death syndrome, and unexpected infant death. Am J Dis Child. 1993;47:1097-100.

15. Krous HF, Nadeau JM, Silva PD, Byard RW. Infanticide: Is its incidence among Postnenatal Infant Deaths increasing? Am J Forensic Medicine Path.2002;23:127-31.

16. Meadows R. Recurrent cot death and suffocation. Arch Dis Child.1989;64:179-80.

17. Meadows R. Unnatural sudden infant death. Arch Dis Child. 1999;80:7-14.

18. Oehmichen M, Gerling I, MeiBner C. Petechiae of the baby's skin as differentiation symptom of infanticide versus SIDS. J Forensic Sci.2000;45:602-7.

19. Reece RM. Fatal child abuse and SIDS: A Critical diagnostic decision. Pediatrics. 1993;91:423-29.

20. Wayte DM. Smothering, suffocation, and cot deaths. Lancet. 1985;1:114.

21. Wolkind S, Taylor EM, Waite AJ. Recurrence of unexpected infant death. Acta Paediatr. 1993;82:873-6.

22. Yukawa N, Carter N, Rutty G, Green MA. Intraalveolar hemorrhage in SIDS: A cause for concern? J Clin Path. 1999;52:581-7. 
23. Bohnert M, Perdekamp MG, Poolak S. Three subsequent infanticides cover up as SIDS. Int J Legal Med. 2004;19:31-4.

24. Kirschner R. The pathology of child abuse. In M. Helfer, R Kempe, R Krugman, (Eds.) The Battered Child (5th ed., pp 248-295). Chicago: University of Chicago Press. 1997.

25. Beckwill JB. Defining the sudden infant death syndrome. Arch Pediatr Adolesc Med. 2003;157:286-90.

26. Emert JL. Families in which two or more cot deaths have occurred. Lancet. 1986;1:3-15.

27. Firstman R, Talan J. The death of innocents. New York: Bantam, 1997.

28. Guntheroth W, Lohmann R, Spiers P. Risk of SIDS in subsequent siblings. J Pediatr. 1990;116:520-4.

29. Hunt CE. SIDS and other causes of infant mortality. Am J Reso Crit Care Med. 2001;164:347-57.

30. Meadows R. Munchausen's syndrome by proxy. The hinterland of child abuse. Lancet. 1977;2:343-5.

31. Pinholster G.SIDS paper triggers a murder change. Science. 1994;264:197-8.

32. DiMaio VJ, DiMaio D. Forensic Pathology. Boca Raton, CRC Press, 2000.

33. Hill R. Multiple sudden Infant Deaths-Coincidence or Beyond Coincidence? Paediatric Perinatal Epid. 2004;18:320-26.

34. Koehler SA, Ladham S, Shakir A, Wecht CH. Simultaneous Sudden Infant Death Syndrome: A proposed definition and a Worldwide Review of Cases. American Journal of Forensic Medicine and Pathology. 2001;22:23-32.

35. Sudden Unexpected Infant Death Case Registry. Available at http://www.cdc.goc/sids/CaseRegistry.htm Accesses 1/24/2013.

36. Pitt SE, Bale EM. Neonaticide, Infanticide, and Filicide: A Review of the Literature, Bill Am Acad Psychiatry Law. 1995;23:375-86.

37. Confessions Are Key to Unlocking SIDS Cases. Andrew Martin, Robert Becker. Chicago Tribune. May 4 1994. Available at http://articles.chicagotribune.com/1994-05-04/news/9405040142_1_ sids-gail-savage-sudden-infant-death-syndrome
38. Was It SIDS or Murder? State Says It Will Prove Mother Smothered Her Child in 1986. Diane Struzzi. Courant September 9, 2001. Available at http:/ / articles. courant.com/2001-09-09/news/0109092175_1_sidsor-murder-smothering-arrest-warrant

39. The Forgotten Children: Justice Undone: Bungled Investigation lets Killer Off. Jane Hansen. Atlanta Journal and the Atlanta Constitution. April 23, 2000. Available at http://www.gahsc.org/terrell/ deaths $2 . h t m l$

40. Light MJ, Sheridan MS. Munchausen syndrome by Proxy and apnea (MBPA)- A Survey of apnea programs. Clinical Pediatrics. 1990;29:162-8.

41. Hall DE, Eubanks L, Meyyazhagan S, Kenney RD, Johnson SC. Evaluation of covert video surveillance on the diagnosis of Munchausen syndrome by Proxy: Lesions from 41 cases. Pediatrics. 2000;105:1305-12.

42. Southall DP, Plunkett MCB, Banks MW, Falkov AF, Samuels MP. Covert video recordings of life threatening chills abuse: Lessons for chills protection. Pediatics. 1997;100:735-60.

43. Steinschneider A. Sudden infant death syndrome. In: Hockleman RA, Blatman S, Brunnel SG, Seidel HM, eds. Principles of Pediatrics: Health Care of the Young. New York, NY: McGraw-Hill. 1978.

44. Pollock HA. Changes in the timing of SIDS deaths in 1989 and 1999: Indirect evidence of low homicide prevalence among reported cases. Paediatric Perinatal Epid. 2006;20:2-13.

45. Emery JL. Child abuse, sudden infant death syndrome, and unexpected infant death. Am J Dis Child. 1993;147:1097-100.

46. Kukull WA, Peterson D. Sudden infant death and infanticide. Am J Epidemiol. 1977;106:485-6.

47. When It's Death by Unnatural Causes: Medicine: Infanticide? Or SIDS New Research and Suspicious Deaths of Babies have Health Officials looking into ways to between determined what-or who-is the Killer. Kathleen Doheny. Los Angel Times. July 16, 1996. Available at http://articles. latimes.com/1996-07-16/news/1s-24487_1_infant-death-syndrome

48. Jay D. Homicide and the Baby-Sitter. Am J For Med. 1998;19:321-3.

Citation: Koehler AS, Applegate MK. Covert homicide: When SIDS is not SIDS, reasons for the missed identification. Paediatrics Today. 2013;9(1):13-23. 